\title{
Prolotherapy injections and eccentric loading exercises for painful Achilles tendinosis: a randomised trial
}

\author{
Michael J Yelland, ${ }^{1}$ Kent R Sweeting, ${ }^{1}$ John A Lyftogt, ${ }^{2}$ Shu Kay Ng, ${ }^{1}$ \\ Paul A Scuffham, ${ }^{1}$ Kerrie A Evans ${ }^{1}$
}

${ }^{1}$ Griffith University, Logan and Gold Coast, Australia ${ }^{2}$ Active Health QEII,

Christchurch, New Zealand

\section{Correspondence to}

Professor Michael J Yelland, School of Medicine, Griffith University Logan Campus, University Drive, Meadowbrook, Queensland 4131, Australia;

m.yelland@griffith.edu.au

Accepted 28 May 2009

\begin{abstract}
Objective To compare the effectiveness and costeffectiveness of eccentric loading exercises (ELE) with prolotherapy injections used singly and in combination for painful Achilles tendinosis.

Design A single-blinded randomised clinical trial. The primary outcome measure was the VISA-A questionnaire with a minimum clinically important change (MCIC) of 20 points

Setting Five Australian primary care centres.

Participants 43 patients with painful mid-portion Achilles tendinosis commenced and 40 completed treatment protocols.
\end{abstract}

Interventions Participants were randomised to a 12-week program of ELE $(n=15)$, or prolotherapy injections of hypertonic glucose with lignocaine alongside the affected tendon $(n=14)$ or combined treatment $(n=14)$.

Main outcome measurements VISA-A, pain, stiffness and limitation of activity scores; treatment costs.

Results At 12 months, proportions achieving the MCIC for VISA-A were $73 \%$ for ELE, 79\% for prolotherapy and $86 \%$ for combined treatment. Mean $(95 \% \mathrm{Cl})$ increases in VISA-A scores at 12 months were 23.7 (15.6 to 31.9) for ELE, 27.5 (12.8 to 42.2) for prolotherapy and 41.1 (29.3 to 52.9) for combined treatment. At 6 weeks and 12 months, these increases were significantly less for ELE than for combined treatment. Compared with ELE, reductions in stiffness and limitation of activity occurred earlier with prolotherapy and reductions in pain, stiffness and limitation of activity occurred earlier with combined treatment. Combined treatment had the lowest incremental cost per additional responder (\$A1539) compared with ELE.

Conclusions For Achilles tendinosis, prolotherapy and particularly ELE combined with prolotherapy give more rapid improvements in symptoms than ELE alone but long-term VISA-A scores are similar.

Trial registration number ACTRN: 12606000179538

Tendinopathy of the Achilles tendon is a frequent cause of pain and disability in people with an active lifestyle. ${ }^{12}$ The condition is one of the most common overuse injuries in sports that repetitively load the Achilles tendon, such as athletics, orienteering, badminton, soccer and volleyball. ${ }^{1-3}$

Achilles tendinosis is the term used when structural changes in the Achilles tendon are observed on ultrasound. Painful Achilles tendinosis most commonly affects the mid portion of the tendon where collagen degeneration and new vessel and nerve formation exist in the absence of inflammation. ${ }^{4-7}$ Mid-portion Achilles tendinosis has proven very difficult to treat, ${ }^{8}$ with reported success rates of $0 \%$ to $36 \%$ for treatments including rest, anti-inflammatory medications, stretching and massage 5910 and from $70 \%$ to $85 \%$ with surgery followed by 4-6 months' rehabilitation. ${ }^{2} 8$ The current reference standard for the conservative treatment of Achilles tendinosis is an intensive programme of eccentric loading exercises (ELE) involving 180 eccentric calf muscle contractions daily for 12 weeks. ${ }^{9} 10$ This has a success rate of $60 \%$ to $100 \% .691112$ Clinical improvement is strongly associated with the disappearance of neovascularisation and structural changes in the tendon after ELE. ${ }^{6}$ Similar clinical and sonographic responses have been shown after the injection of polidocanol into the neovessels, but this requires ultrasound guidance and specialised ultrasonographic skill and equipment. ${ }^{13} 14$ Prolotherapy is a simpler injection treatment, not requiring ultrasound, where a solution of hypertonic glucose and local anaesthetic is injected alongside the painful areas of the tendon ${ }^{15}$ with the aim of stimulating inflammation followed by collagen deposition. ${ }^{16}$ A study of glucose/lignocaine injections versus saline injections into injured rat knee ligaments has shown additional thickening of healing ligaments but no differences in biomechanical properties. ${ }^{17}$ Prolotherapy also aims to reverse the neovascularisation accompanying tendinosis, but this effect is still speculative. Nonetheless, there is level 4 evidence of rapid and sustained reductions in pain and disability with courses of prolotherapy for both hip adductor tendinopathy ${ }^{15}$ and Achilles tendinopathy. ${ }^{18} 19$ Given that prolotherapy injections require much less time and patient effort than ELE, prolotherapy may be an attractive treatment alternative for people with Achilles tendinopathy.

There is evidence of a synergistic effect of ELE when combined with other treatments, such as low-level laser therapy ${ }^{20}$ and glyceryl trinitrate patches. ${ }^{21}$ This phenomenon has not been tested with prolotherapy. Thus, we performed a randomised clinical trial (RCT) of ELE, and prolotherapy injections were used singly and in combination for painful mid-portion Achilles 
tendinosis to examine differences in clinical and functional outcomes and treatment costs.

\section{METHODS}

\section{Setting and participants}

The trial was conducted in Australia across five centres. The inclusion criteria were diagnosis of unilateral or bilateral midportion Achilles tendinosis with pain between 2 and $7 \mathrm{~cm}$ proximal to the calcaneal attachment in adults $>18$ years with activity-related pain for at least 6 weeks. The clinical severity of the tendinosis had to yield a score on the Victorian Institute of Sport Assessment-Achilles (VISA-A) ${ }^{2}$ of $<80$ of a maximum of 100 for participants involved in sport and $<70$ of 90 for people not involved in sport. The exclusion criteria were previous steroid or prolotherapy injections or surgery to the affected tendon, previous completion of $>50 \%$ of the Achilles ELE protocol and any allergies or medical conditions that might limit completion of trial treatments.

The sample size was determined by the available resources and the slow recruitment rate rather than by an a priori sample size calculation. The participants were recruited from April 2006 to June 2007 via referrals from health professionals and through advertising in newspapers, brochures and a website. Applicant eligibility was assessed by phone interview followed by a clinical assessment and ultrasound with Doppler. Imaging protocols and reporting procedures were standardised across all the trial centres. The participants gave written consent at enrolment and were randomised to undertake ELE, prolotherapy injections or a combination of ELE and prolotherapy injections (combined treatment), using a computer-generated randomisation schedule with a block size of six. The randomisation schedule was generated and administered by telephone independently by the National Health and Medical Research Council Clinical Trials Centre in Sydney, Australia. Ethics approval for the study was given by the Griffith University Human Research Ethics Committee in Brisbane, Australia.

\section{Patient evaluation}

The primary outcome measure was the VISA-A questionnaire, an aggregate measure that comprised eight questions grading the domains of pain, stiffness, function in daily living and sporting activity. It has good reliability, validity and stability. ${ }^{2}$ The VISA-A questionnaire was administered during the clinical assessment at baseline and via telephone interview at 6 weeks and 3, 6 and 12 months. The criterion for success of treatment was set at an increase of 20 points in the VISA-A score. This was the minimum clinically important change (MCIC) estimated by investigators who estimate for the VISA-A Patella questionnaire used for patellar tendinopathy. ${ }^{22}$ We used this only because there were no published estimates of the MCIC for the VISA-A questionnaire. We defined fully recovered as attaining a score of $\geq 90$.

Secondary outcomes included seven-point Likert scales for treatment satisfaction (from extremely dissatisfied to extremely satisfied $)^{23}$ and the Patient Global Impression of Change (PGIC) scale (from very much worse to very much better) ${ }^{2425}$ and 0 to 10 scales for worst pain in the last week, usual morning stiffness and limitation of usual activities. In the absence of MCIC values for these outcomes for Achilles tendinosis, we used MCIC values for other musculoskeletal conditions, that is, 1.75 for pain, ${ }^{26} 1.75$ for stiffness ${ }^{27}$ and 2.0 for limitation of activities, ${ }^{28}$ to assess the clinical importance of differences between groups. Achievement of two treatments goals, predetermined by each participant, was assessed. Use of other treatments was also recorded. All direct treatment costs related to the Achilles tendinosis from the preceding 3 months were recorded, including costs for general practitioner and specialist visits, outpatient visits with allied health professionals (eg, physiotherapists and podiatrists), aids and appliances and medications. These costs represented the costs to both the government and the patient. The cost of the interventions (ELE, prolotherapy and combined treatment) were calculated from the Australian Medicare Benefits Schedule with no patient copayment. ${ }^{29}$ Secondary outcome measures were administered at baseline (with the exception of the satisfaction and PGIC scales) and at 3 and 6 and 12 months. All outcome measures were administered by an assessor blinded to group allocation.

\section{Treatment protocols}

Each treatment was applied according to a standardised protocol described in a trial manual and adopted by all treating practitioners.

\section{Eccentric loading exercises}

The participants were instructed by a doctor or podiatrist in the ELE protocol described by Alfredson et al. ${ }^{9}$ The exercises are performed twice daily in three sets of 15 repetitions with the knee straight and three sets of 15 repetitions with the knee bent for a period of 12 weeks. The participants are told that the exercises may be painful but not to exceed an intensity of $4 / 10$. As the pain eases over time, load is progressively increased by adding weights to a backpack. The participants had an initial training session and then reviews at 3, 6 and 12 weeks to check technique and progress. Written instructions for the exercises were supplied, and the participants kept a diary to document exercise load and compliance. Compliance was categorised by the percentage of the protocol performed. ${ }^{30}$

\section{Prolotherapy injections}

A doctor injected tender points in the subcutaneous tissues adjacent to the affected tendon with a solution consisting of $20 \%$ glucose $/ 0.1 \%$ lignocaine $/ 0.1 \%$ ropivacaine weekly for four to 12 treatments, using the technique described by Lyftogt. ${ }^{18}$ The tender points were most commonly the anterolateral and anteromedial margins of the tendon and on the most posterior aspect of the tendon $2-7 \mathrm{~cm}$ from the calcaneus attachment. At each point, $0.5-1 \mathrm{ml}$ of solution was used to a maximum total of $5 \mathrm{ml}$. The number of treatments was determined by the time it took to reach a pain-free activity or until the participant requested to cease treatment.

\section{Combined treatment}

The protocols for ELE and prolotherapy injections were applied concurrently for this group. The participants in all the three groups were encouraged to gradually increase their activity levels while their symptoms reduced. They were discouraged from taking any anti-inflammatory medication but were allowed to take analgesics for pain relief.

\section{Statistical analysis}

Data analysis was performed blinded to group allocation on an intention-to-treat basis. Missing data occur intermixed with non-missing values (intermittent missing values), which are filled in by assuming a linear trend between the observed measurements. The proportion of missing final outcome measurements is small $(<3 \%$ for the primary outcome measure), and they are imputed by carrying the last value 
forward method. ${ }^{31}$ The method assumes that there was no further change for an individual since the last available measurement. This assumption is not plausible in cases where the patients withdraw because of the treatment received. However, as the missing final measurements appear in the ELE and prolotherapy treatment groups only, the adoption of the last value forward method is conservative ${ }^{32}$ for estimating the effect size of the combined treatment group. The SAS version 9.1.3 software (PROC GENMOD) was used to analyse the longitudinal data using the generalized estimating equation (GEE), with a first-order autoregressive relationship (AR[1]) working correlation structure to account for within-patient correlation for repeated measurements. ${ }^{33}$ With the GEE, a normal distribution with an identity link was used for scale variable outcomes, whereas a binomial or multinomial distribution with a logit link was used for categorical variable outcomes. The assumption of normality within the framework of GEE was checked for scale variable outcomes. The effects of time (within-group differences), treatment (between-group differences) and treatment by time interaction (between-group differences over time) were included in all the models and assessed using the Wald $\chi^{2}$ test. Demographic and clinical variables at baseline were compared between treatment groups to assess the effectiveness of randomisation.

\section{Economic analysis}

A cost-effectiveness analysis for patient achieving MCIC at 12 months (responders) was performed. The ELE was the primary comparator, and the incremental costs and the additional benefit of prolotherapy and combined treatment was calculated. The incremental cost-effectiveness ratio (ICER; ie, additional costs divided by the additional benefit) is then used to determine the most efficient use of resources. ${ }^{33}$ A sensitivity analysis was undertaken to address any variation in the costs of the intervention, health care use and uncertainty around the effectiveness of the intervention. All the costs are reported based on the 2008 value of the Australian dollar currency (\$A1 €0.50).

\section{RESULTS \\ Patients}

Figure 1 demonstrates the flow of participants through the study. A total of 145 people were interviewed by phone, from which 60 were clinically examined. A clinical diagnosis of mid-portion Achilles tendinopathy was made in 50 people. Of these, 43 had ultrasound findings of mid-portion tendinosis and consented to be in the study. A total of 15 participants were allocated to the ELE group, with 14 participants in each of the prolotherapy and combined treatment groups. By the end of the study, three participants had discontinued treatment, all of whom were from the ELE group. One participant had a heart attack, one discontinued because of time restraints and one sustained an unrelated injury during the study. One participant who was assigned to the prolotherapy group did not begin treatment because of work commitments.

The demographic and clinical characteristics of the three groups were similar, although the median duration of pain

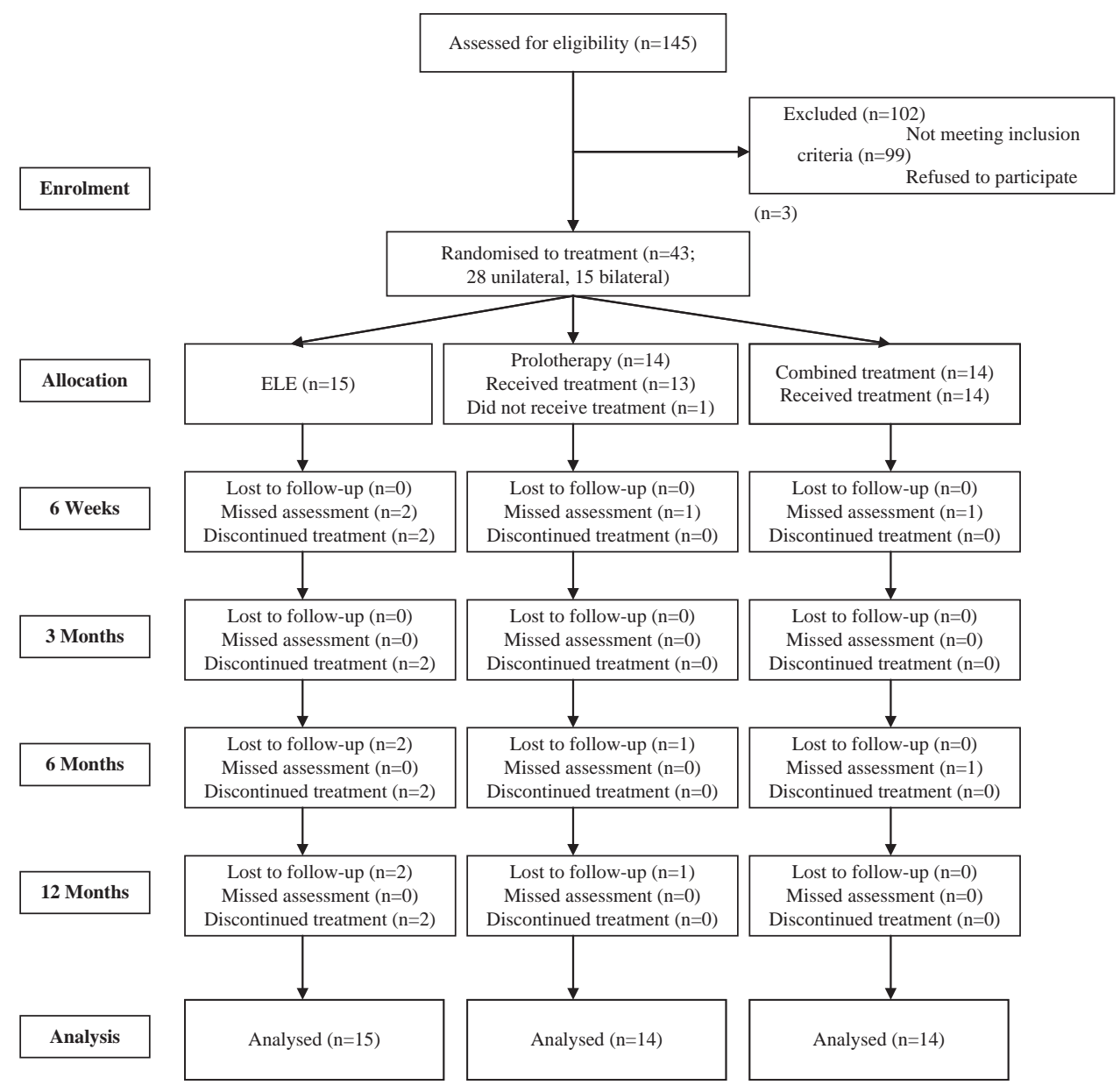

Figure 1 CONSORT flow diagram of trial applicants and participants. 
and the baseline VISA-A score in the combined treatment group were somewhat less than those in the exercise and prolotherapy groups (table 1).

\section{Treatment}

The mean (SD) number of visits per group was 3.3 (1.9) for ELE, 9.5 (2.8) for prolotherapy and 8.7 (2.9) for the combined treatment. The mean (SD) number of injections per visit was 4.4 (1.7) for the prolotherapy group and 4.4 (1.6) for the combined treatment group.

In the ELE group, seven participants returned exercise diaries. Of these, exercise compliance was moderate $(25 \%$ to $50 \%$ ) for one, good (50\% to $75 \%$ ) for two and excellent ( $>75 \%)$ for four. In the combined group, nine participants returned exercise diaries. Of these, exercise compliance was moderate for one, good for three and excellent for five.

One adverse event was reported in the trial. A participant in the ELE group had a partial calf tear while playing tennis. An independent sports physician did not attribute this to the ELE programme.

\section{VISA-A scores}

There were significant improvements in VISA-A scores at all times compared with those at baseline for all the groups $(p<0.0005$; fig. 2). These changes exceeded the MCIC threshold of 20 points by 3 months in all the groups. The mean (95\% confidence interval [CI]) increase in VISA-A scores at 12 months was 23.7 (15.6 to 31.9) for ELE, 27.5 (12.8 to 42.2) for prolotherapy and 41.1 (29.3 to 52.9 ) for combined treatment. The increases from baseline for the ELE group were significantly less than for the combined treatment group at 6 weeks (difference $-11.7 ; 95 \%$ Wald CI-3.5 to $-19.9 ; \mathrm{p}=0.005$ ) and 12 months (difference -17.3 ; 95\% Wald CI -4.8 to -29.9 ; $\mathrm{p}=0.007)$. There were significant differences between treatment groups over time (Wald $\chi^{2}$ statistics 23.7 ; $d f=8 ; p=0.003$ ).

The percentage within each group achieving the MCIC of 20 points in VISA-A scores from baseline increased significantly over time $(p<0.0005)$ and was generally highest for the combined treatment group (table 2). However, there were no significant differences between groups or between groups over time.

The percentage achieving full recovery with a VISA-A score of $\geq 90$ at 12 months was $53 \%$ for ELE, $71 \%$ for prolotherapy and $64 \%$ for combined treatment, but there were no significant differences between groups over time.

\section{Pain, stiffness and limitation of activity scores}

There were significant decreases in pain, stiffness and limitation of activity scores over time within all the groups $(p<0.0005$, fig. 3). The decreases in pain scores from baseline for ELE were significantly less by a clinically important difference than for prolotherapy at 6 months (difference 2.3; $95 \%$ Wald CI 0.3 to $4.4 ; \mathrm{p}=0.028$ ) and for combined treatment at 12 months (difference 2.6; 95\% Wald CI 0.4 to 4.8; $\mathrm{p}=0.02)$. The difference between the treatment groups over time, however, was not significant overall (Wald $\chi^{2}$ statistics 13.2; $\mathrm{df}=8 ; \mathrm{p}=0.105)$. The decreases in stiffness scores from baseline for ELE were significantly less by a clinically important difference than for prolotherapy at 6 months (difference 1.6; 95\% Wald CI0.3 to 3.0; $\mathrm{p}=0.019$ ) and for combined treatment at 6 weeks (difference 2.8; 95\% Wald CI 0.8 to 4.8; $\mathrm{p}=0.007$ ) and 12 months (difference $3.4 ; 95 \%$ Wald CI1.2 to 5.6; $\mathrm{p}=0.002)$. The difference between the groups over time was marginally significant (Wald $\chi^{2}$ statistics 16.0; $\mathrm{df}=8$; $p=0.043)$. However, it is cautioned that the significance must
Table 1 Demographic and clinical characteristics and trial treatment details by treatment group

\begin{tabular}{|c|c|c|c|}
\hline Variable & ELE (n=15) & $\begin{array}{l}\text { Prolotherapy } \\
(n=14)\end{array}$ & $\begin{array}{l}\text { Combined } \\
\text { treatment } \\
(n=14)\end{array}$ \\
\hline Median (IOR) & & & 0 \\
\hline Age & $46(40-58)$ & $48(41-54)$ & $46(40-57)$ \\
\hline Duration of pain, mo & $21(14.3-51)$ & $24(7-78)$ & $6(4.5-22)$ \\
\hline \multicolumn{4}{|l|}{ n (\%) } \\
\hline $\begin{array}{l}\text { Participates in } \\
\text { physical activity }\end{array}$ & $14(93.3)$ & $14(100)$ & $12(85.7)$ \\
\hline \multicolumn{4}{|l|}{ Side of pain, $n(\%)$} \\
\hline Left & $6(40.0)$ & $7(50.0)$ & $4(28.6)$ \\
\hline Right & $4(26.7)$ & $2(14.3)$ & $5(35.7)$ \\
\hline Bilateral (left worse) & $1(6.7)$ & $2(14.3)$ & $2(14.3)$ \\
\hline Bilateral (right worse) & $4(26.7)$ & $3(21.4)$ & $3(21.4)$ \\
\hline Received previous treatment & $14(93.3)$ & $14(100)$ & $12(85.7)$ \\
\hline
\end{tabular}

Table 2 Number (\%) of participants achieving the minimum clinically important increase of 20 points or more in VISA-A scores from baseline over time by treatment group

\begin{tabular}{lllll}
\hline Treatment group & $\mathbf{6} \mathbf{w k}, \mathbf{n}(\%)$ & $\mathbf{3} \mathbf{~ m o}, \mathbf{n}(\%)$ & $\mathbf{6} \mathbf{~ m o}, \mathbf{n}(\%)$ & $\mathbf{1 2 ~} \mathbf{~ m o}, \mathbf{n}(\%)$ \\
\hline ELE $(\mathrm{n}=15)$ & $3(20)$ & $8(53)$ & $7(47)$ & $11(73)$ \\
Prolotherapy $(\mathrm{n}=14)$ & $5(36)$ & $7(50)$ & $11(79)$ & $11(79)$ \\
$\begin{array}{l}\text { Combined treatment } \\
(\mathrm{n}=14)\end{array}$ & $7(50)$ & $8(57)$ & $10(71)$ & $12(86)$
\end{tabular}

be adjusted for multiple testing of the three effects (withingroup, between-group and between-group over time). The decreases in limitation of activity scores from baseline for ELE were significantly less by a clinically important difference than for combined treatment at 6 weeks (difference 2.2; 95\% Wald CI0.3 to 4.1; $\mathrm{p}=0.024)$. The difference between groups over time, however, was not significant overall (Wald $\chi^{2}$ statistics $13.5 ; \mathrm{df}=8 ; \mathrm{p}=0.096$ ).

It should be noted that for each of the above-mentioned clinically important differences between the groups, the CIs are very wide; so much smaller differences between the groups cannot be ruled out.

\section{Satisfaction and global change ratings}

There were no significant differences in satisfaction ratings or in PGIC ratings between the groups over time. The percentage reporting "satisfaction" or "extreme satisfaction" with treatment at 12 months was $50 \%$ for ELE, $69 \%$ for prolotherapy and $71 \%$ for the combined treatment. The percentage reporting a PGIC of "much improved" or "very much improved" at 12 months was $75 \%$ for ELE, $85 \%$ for prolotherapy and $64 \%$ for combined treatment.

\section{Economic analysis}

The costs of the specific interventions made up the largest proportion of total costs accounting for $65 \%, 94 \%$ and $73 \%$ of the total costs in the ELE, prolotherapy and combined treatment groups, respectively. Compared with ELE, prolotherapy cost an additional $\$ 90$ in total and combined treatment cost $\$ 191$ (table 3). For those additional costs, an additional 5.2\% of the participants achieved a $\geq 20$-point improvement in VISA-A score from prolotherapy at 12 months, whereas for the combined treatment, an additional $13 \%$ achieved this response. From the ICERs, it is apparent that combined treatment offers the best value for money (ie, the additional cost per responder is less than prolotherapy alone). 
Table 3 Costs and cost-effectiveness per participant by treatment group

\begin{tabular}{llll}
\hline & ELE, \$A & Prolotherapy, \$A & $\begin{array}{l}\text { Combined } \\
\text { treatment, \$A }\end{array}$ \\
\hline $\begin{array}{l}\text { Intervention costs } \\
\text { Medicare costs* }\end{array}$ & 259 & 460 & \\
Non-trial costs & & & 433 \\
Medicare costs* & 46 & 25 & \\
GP and specialist visits ${ }^{*}$ & 46 & 2 & 0 \\
Allied health professionals & 25 & 0 & 9 \\
Other costs & 18 & 0 & 79 \\
Pharmaceuticals & 6 & 3 & 61 \\
Total non-trial costs & 141 & 30 & 8 \\
Total cost per patient & 400 & 490 & 158 \\
Responders (at 12 mo) & 0.733 & 0.786 & 591 \\
Incremental analysis & & & 0.857 \\
(vs ELE alone) & & & \\
Additional costs, \$A & & 90 & 191 \\
Additional responders & & 0.052 & 0.124 \\
ICER (\$A /responder) & & 1716 & 1539 \\
Sensitivity analysis & & ICER, \$A & ICER, \$A \\
+20\% intervention costs & & 3473 & 2238 \\
-20\% intervention costs & & Dominates $\ddagger$ & 839 \\
+ +20\% non-trial costs & & 1831 & 1793 \\
-20\% non-trial costs & & 1602 & 1284 \\
+10\% responders & & 687 & 714 \\
-10\% responders & & Dominated§ & 5001 \\
\hline
\end{tabular}

* Includes assessment and treatment. These costs are fully covered by Medicare, the government health insurance scheme.The general practitioners and specialist visit costs are in addition to those reimbursed by Medicare and are incurred by the patient.

tA proportion of those costs may be reimbursed to the patient depending on the level of cover of any health insurance they may have.

$\ddagger$ A strategy dominates where greater health benefits are obtained at a lower cost than the comparator.

$\S A$ strategy is said to be dominated where fewer health benefits are obtained at a higher cost than the comparator.

These results were most sensitive to changes in the proportion of responders-for example, when this is reduced in the prolotherapy group by $10 \%$, ELE is superior (lower cost and more responders) to prolotherapy. Reducing the cost of prolotherapy by $20 \%$ resulted in prolotherapy being superior to ELE. Reducing the cost of combined treatment by $20 \%$ resulted in a $45 \%$ reduction in the ICER (and vice versa for increases in the intervention costs). Varying the costs for healthcare by $\pm 20 \%$ over the trial and follow-up periods made relatively little difference to the ICERs.

\section{DISCUSSION}

This is the first trial to compare prolotherapy injections with the reference standard treatment of ELE. It offers a comprehensive picture of treatment inputs, clinical outcomes and costs to inform clinical decision making. Although the long-term outcomes summarised by VISA-A scores are similar, there are some differences in treatment inputs and reductions in symptoms. The ELE offers a low cost, self-management programme requiring considerable effort by the patient but with a slower reduction in symptoms. Prolotherapy is a relatively simple office procedure that offers a more time-efficient approach to treatment and a better reduction in pain and stiffness at 6 months but involves the pain of multiple injections, more doctor visits and a higher cost. Combined treatment requires the greatest input from the patient, clinician and health budgets but offers better reductions in pain and limitation of activity
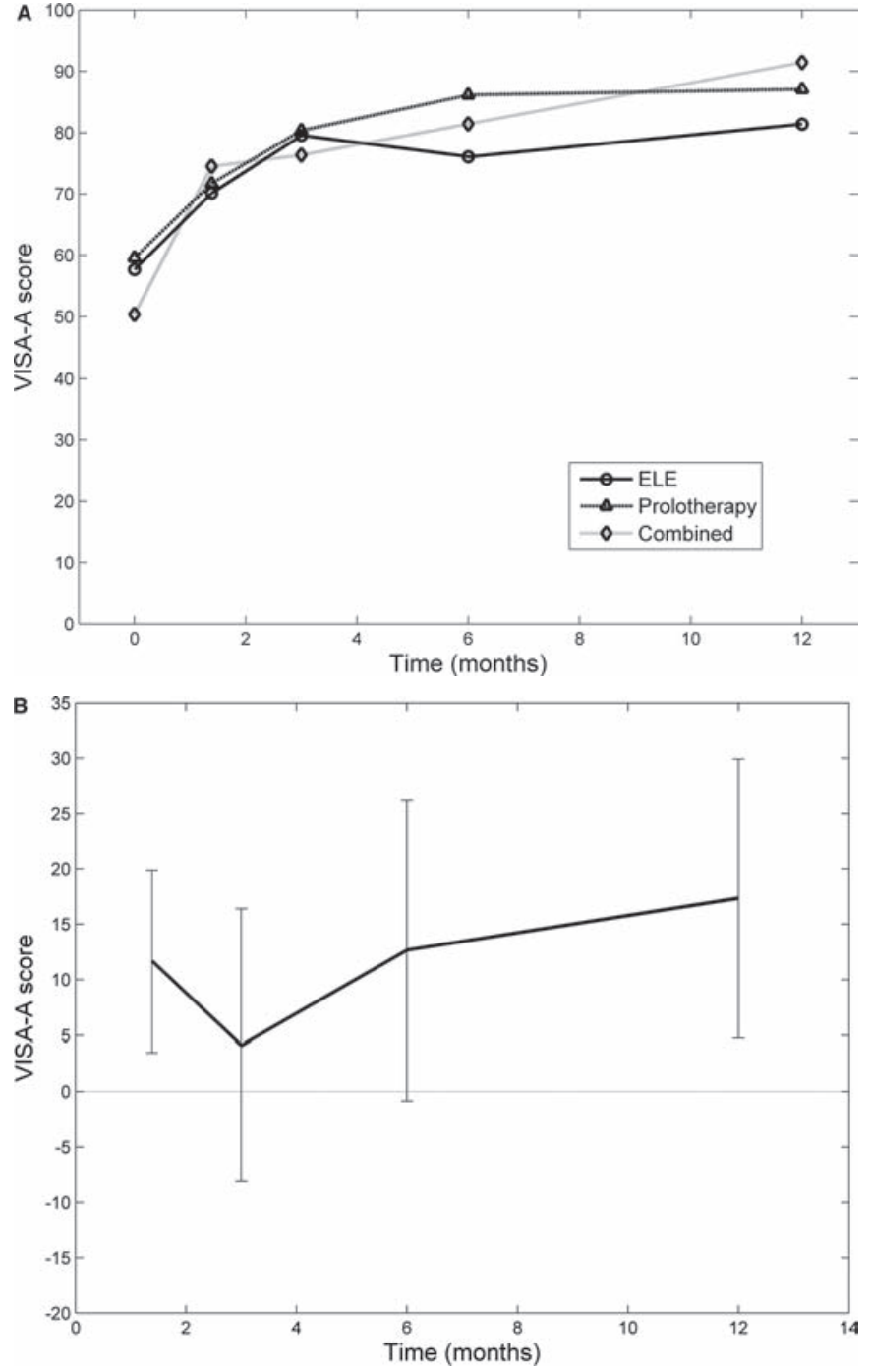

Figure 2 (A) Mean VISA-A scores by treatment group over 12 months; (B) Treatment differences ('Combined' relative to 'ELE') with associated $95 \%$ Cls.

at 6 weeks and in pain and stiffness at 12 months and the lowest additional costs per additional responder. This is consistent with other studies comparing single treatment with combined treatment. ${ }^{2021}$ The results could indicate some attention bias for those undergoing prolotherapy or combined prolotherapy, as this required an average of 9.5 and 8.7 treatments, respectively, compared with 3.3 visits for the ELE participants.

Strengths of this study include a comprehensive assessment of outcomes over 12 months, successful randomisation and blinding procedures, and a full economic evaluation. The blinded assessor was able to correctly identify the treatment group for 12 (28\%) of 43 participants; he did not know in 15 of $43(35 \%)$ cases, and was incorrect for 16 of $43(37 \%)$ participants.

Although a trial design incorporating a no-treatment or at least a waiting list control group was desirable, we decided against this, believing that it would inhibit recruitment, which is already difficult enough in an RCT. We can find only one RCT on Achilles tendinopathy with a wait-and-see group. ${ }^{34}$ The outcomes for this approach were inferior to ELE in that trial and ELE in our trial.

To our knowledge, the only one other RCT of prolotherapy for tendinosis was for lateral epicondylosis, where 

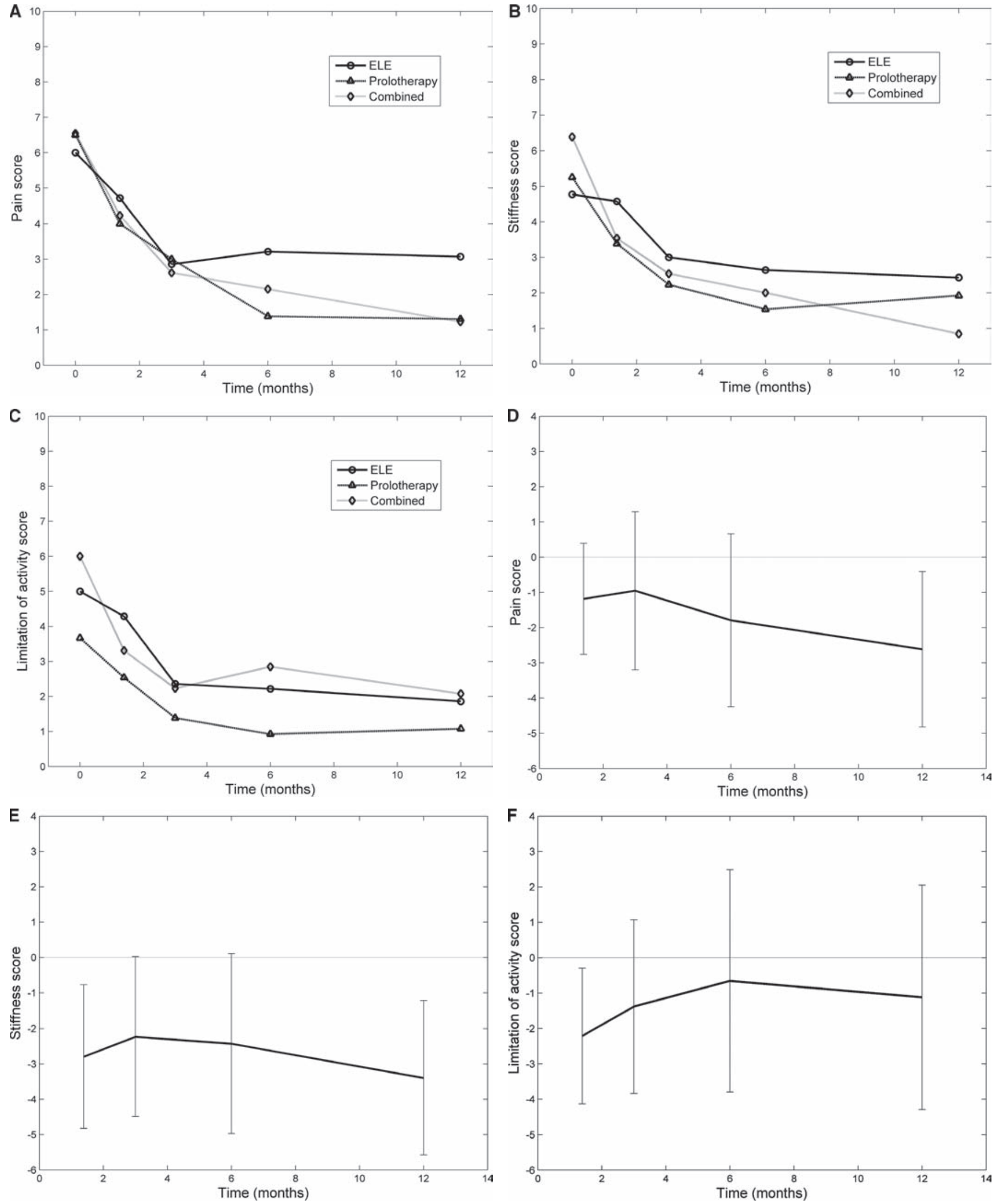

Figure 3 Mean scores for (A) pain, (B) stiffness and (C) limitation of activity for treatment groups over 12 months. Treatment differences ('Combined' relative to 'ELE') with associated $95 \%$ Cls for (D) pain, (E) stiffness and (F) limitation of activity.

injections of sodium morrhuate, glucose and local anaesthetic injections were more effective than saline injections. ${ }^{35}$ This is the first RCT of prolotherapy for Achilles tendinosis. The improvements in pain at 6-12 months were inferior to those in uncontrolled observational studies of prolotherapy. ${ }^{18}$ 19 This could be because of our use of more comprehensive outcome measures, differences in patient populations, differences in technique or the greater experience of the treating 
doctors in previous studies. In the Results section, we have compared the treatment effects over time for the primary and the three secondary outcome measures separately. It should be noted that the overall experiment-wise type 1 error rate will be inflated when comparisons arisen from multiple outcome measures are considered. ${ }^{36}$

This trial provides valuable information for future treatment trials of Achilles tendinosis. Our concurrent collection of VISA-A scores and PGIC ratings allows an estimate of the MCIC for the VISA-A score. The best method for this is the sensitivity-specificity approach, which estimates the difference in VISA-A change scores between the much improved/ very much improved group and the remainder. ${ }^{37} 38$ The MCIC is the threshold of this difference that discriminates between the two groups with equal sensitivity and specificity. Using this method, the MCIC value for the VISA-A questionnaire was 28 , somewhat greater than the value used as the benchmark for success in this trial. At an MCIC of 28, the response rates at 12 months become $47 \%$ for ELE and $72 \%$ for combined treatment, a difference of $25 \%$. This difference reduces the ICER for combined treatment to only $\$ 764$ per additional responder.

\section{CONCLUSIONS}

In physically active people with painful Achilles tendinosis, most patients show sustained improvements with ELE and prolotherapy used singly or in combination, but that improvements in mean symptom scores occur earlier with prolotherapy and, particularly, with combined treatment than with ELE alone. Improvements in mean VISA-A scores are greater with combined treatment than with ELE alone, but the proportions achieving clinically important improvements in VISA-A scores are similar for all groups. Combined treatment involves a small increase in cost and temporary discomfort from treatment. A larger clinical trial is needed to further explore these findings.

\section{What is already known on this topic}

In painful Achilles tendinosis,

- ELEs are effective but the addition of other treatments may enhance their effect.

- There is level 4 evidence that prolotherapy injections are effective.

- The relative effectiveness of ELEs and prolotherapy used singly and in combination has not been established.

\section{What this study adds}

- In painful Achilles tendinosis, prolotherapy and, particularly, ELEs combined with prolotherapy injections give more rapid improvements in symptoms than ELEs alone, but long-term summary outcomes are similar.

- Cost-effectiveness analysis shows that ELEs was the lowest cost treatment, but when combined with prolotherapy, the cost per additional responder was exceptionally good value for money.
Acknowledgements The authors thank Michael Yelland, Michael Oei, Vic Wilk and Scott Masters (Musculoskeletal Medicine Practitioners) for assessing and treating the participants in all the three groups; Lyndon Homeming (Podiatrist) for treating the participants in the ELE group; Mark Young, Kent Sweeting (Podiatrist) and Nicole Magner (Physiotheraist) for assessing the applicants; Mark Young (Sports Physician) for independently reviewing the participants who failed to improve; Natasha Morton, Elizabeth Pyke, Gemma Hynard and Xanthe Golenko for administrative assistance and Queensland X-Ray for standardising the ultrasound protocols.

Funding The trial was funded by grants from the Musculoskeletal Research Foundation of Australia, the Australian Podiatry Education and Research Foundation and the Griffith University Office of Research. Kent Sweeting was supported by a Primary Health Care Research, Evaluation and Development research fellowship. The funding bodies had no role in the study design; in the collection, analysis and interpretation of data; in the writing of the report or in the decision to submit the paper for publication.

Competing interests None.

Ethics approval This study was conducted with the approval of the Griffith University Human Research Ethics Committee.

Provenance and peer review Not commissioned; externally peer reviewed.

Patient consent Obtained.

\section{REFERENCES}

1. Paavola M, Kannus P, Järvinen TA, et al. Achilles tendinopathy. J Bone Joint Surg Am 2002;84-A:2062-76.

2. Robinson JM, Cook JL, Purdam C, et al; Victorian Institute Of Sport Tendon Study Group. The VISA-A questionnaire: a valid and reliable index of the clinical severity of Achilles tendinopathy. Br J Sports Med 2001;35:335-41.

3. Kujala UM, Sarna S, Kaprio J. Cumulative incidence of achilles tendon rupture and tendinopathy in male former elite athletes. Clin J Sport Med 2005; 15:133-5.

4. Ohberg L, Lorentzon R, Alfredson H. Neovascularisation in Achilles tendons with painful tendinosis but not in normal tendons: an ultrasonographic investigation. Knee Surg Sports Traumatol Arthrosc 2001;9:233-8.

5. Maffulli N, Kenward MG, Testa V, et al. Clinical diagnosis of Achilles tendinopathy with tendinosis. Clin J Sport Med 2003;13:11-15.

6. Ohberg L, Alfredson $\mathrm{H}$. Effects on neovascularisation behind the good results with eccentric training in chronic mid-portion Achilles tendinosis? Knee Surg Sports Traumatol Arthrosc 2004;12:465-70.

7. Alfredson $\mathbf{H}$, Ohberg L, Forsgren S. Is vasculo-neural ingrowth the cause of pain in chronic Achilles tendinosis? An investigation using ultrasonography and colour Doppler, immunohistochemistry, and diagnostic injections. Knee Surg Sports Traumatol Arthrosc 2003;11:334-8.

8. Khan KM, Cook JL, Taunton JE, et al. Overuse tendinosis, not tendinitis. Phys Sports Med 2000;28:38-44

9. Alfredson H, Pietilä T, Jonsson P, et al. Heavy-load eccentric calf muscle training for the treatment of chronic Achilles tendinosis. Am J Sports Med 1998;26:360-6.

10. Cook JL, Khan KM, Purdam C. Achilles tendinopathy. Man Ther 2002;7:121-30

11. Mafi N, Lorentzon R, Alfredson H. Superior short-term results with eccentric calf muscle training compared to concentric training in a randomized prospective multicenter study on patients with chronic Achilles tendinosis. Knee Surg Sports Traumatol Arthrosc 2001;9:42-7.

12. Silbernagel KG, Thomeé $R$, Thomeé $P$, et al. Eccentric overload training for patients with chronic Achilles tendon pain — a randomised controlled study with reliability testing of the evaluation methods. Scand J Med Sci Sports 2001;11:197-206.

13. Ohberg L, Alfredson H. Ultrasound guided sclerosis of neovessels in painful chronic Achilles tendinosis: pilot study of a new treatment. Br J Sports Med 2002:36:173-5; discussion 176-7.

14. Alfredson $\mathbf{H}$, Ohberg L. Neovascularisation in chronic painful patellar tendinosis - promising results after sclerosing neovessels outside the tendon challenge the need for surgery. Knee Surg Sports Traumatol Arthrosc 2005:13:74-80.

15. Topol GA, Reeves KD, Hassanein KM. Efficacy of dextrose prolotherapy in elite male kicking-sport athletes with chronic groin pain. Arch Phys Med Rehabil 2005;86:697-702.

16. Banks AR. A rationale for prolotherapy. J Orthop Med 1991;13:54-9.

17. Jensen KT, Rabago DP, Best TM, et al. Response of knee ligaments to prolotherapy in a rat injury model. Am J Sports Med 2008; 36:1347-57.

18. Lyftogt $\mathbf{J}$. Prolotherapy and Achilles tendinopathy: a prospective pilot study of an old treatment. Australas Musculoskel Med 2005;10:16-19.

19. Maxwell NJ, Ryan MB, Taunton JE, et al. Sonographically guided intratendinous injection of hyperosmolar dextrose to treat chronic tendinosis of the Achilles tendon: a pilot study. AJR Am J Roentgenol 2007;189:W215-20. 
20. Stergioulas A, Stergioula M, Aarskog R, et al. Effects of low-level laser therapy and eccentric exercises in the treatment of recreational athletes with chronic achilles tendinopathy. Am J Sports Med 2008;36:881-7.

21. Paoloni JA, Appleyard RC, Nelson J, et al. Topical glyceryl trinitrate treatment of chronic noninsertional achilles tendinopathy. A randomized, double-blind, placebo-controlled trial. J Bone Joint Surg Am 2004;86-A:916-22.

22. Young MA, Cook JL, Purdam CR, et al. Eccentric decline squat protocol offers superior results at 12 months compared with traditional eccentric protocol for patellar tendinopathy in volleyball players. Br J Sports Med 2005;39:102-5.

23. Gordon S, Ameen V, Bagby B, et al. Validation of irritable bowel syndrome Global Improvement Scale: an integrated symptom end point for assessing treatment efficacy. Dig Dis Sci 2003;48:1317-23.

24. Goldsmith CH, Boers M, Bombardier C, et al. Criteria for clinically important changes in outcomes: development, scoring and evaluation of rheumatoid arthritis patient and trial profiles. OMERACT Committee. J Rheumatol 1993;20:561-5.

25. Juniper EF, Guyatt GH, Willan A, et al. Determining a minimal important change in a disease-specific Quality of Life Questionnaire. J Clin Epidemiol 1994;47:81-7.

26. Farrar JT, Young JP Jr, LaMoreaux L, et al. Clinical importance of changes in chronic pain intensity measured on an 11-point numerical pain rating scale. Pain 2001;94:149-58.

27. Bellamy N, Carr A, Dougados M, et al. Towards a definition of "difference" in osteoarthritis. J Rheumatol 2001;28:427-30.

28. Westaway MD, Stratford PW, Binkley JM. The patient-specific functional scale: validation of its use in persons with neck dysfunction. J Orthop Sports Phys Ther 1998;27:331-8.
29. Australian Government Department of Health and Ageing. MBS Online. http://www.health.gov.au/mbsonline (accessed 10 Nov 2008).

30. de Vos RJ, Weir A, Visser RJA, et al. The additional value of a night splint to eccentric loading exercises in chronic midportion Achilles tendinopathy: a randomised controlled trial. Br J Sports Med 2007:41:e5.

31. Bennett DA. How can I deal with missing data in my study? Aust N Z J Public Health 2001;25:464-9.

32. Liang KY, Zeger SL. Longitudinal data analysis using generalised linear models Biometrika 1986;73:13-22.

33. Drummond MF, Sculpher MJ, Torrance GW, et al. Methods for the economic evaluation of health care programmes, 3rd edn. Oxford: Oxford University Press, 2005.

34. Rompe JD, Nafe B, Furia JP, et al. Eccentric loading, shock-wave treatment, or a wait-and-see policy for tendinopathy of the main body of tendo Achillis: a randomized controlled trial. Am J Sports Med 2007:35:374-83.

35. Scarpone M, Rabago DP, Zgierska A, et al. The efficacy of prolotherapy for lateral epicondylosis: a pilot study. Clin J Sport Med. 2008 May;18:248-54.

36. Aickin M, Gensler $\mathrm{H}$. Adjusting for multiple testing when reporting research results: the Bonferroni vs Holm methods. Am J Public Health 1996;86:726-8.

37. Copay AG, Subach BR, Glassman SD, et al. Understanding the minimum clinically important difference: a review of concepts and methods. Spine J 2007; 7:541-6.

38. Pool JJ, Ostelo RW, Hoving JL, et al. Minimal clinically important change of the Neck Disability Index and the Numerical Rating Scale for patients with neck pain. Spine 2007;32:3047-51.

39. CONSORT Group. CONSORT flow diagram. http://www.consort-statement.org (accessed Nov 2008). 